\title{
Tramas en la construcción de la intersectorialidad para atender la primera infancia
}

\author{
Lorena Ceballos-Higuita, Mg. ${ }^{2}$ \\ Universidad de Antioquia, Colombia \\ Gabriel Jaime Otálvaro-Castro, Ph.D.' \\ Universidad del Antioquia, Colombia
}

lore861024@gmail.com

\section{Resumen (analítico)}

Se presentan los resultados de una investigación cualitativa realizada desde una perspectiva interpretativa-histórica, para comprender la construcción de relaciones intersectoriales en el programa de atención a la primera infancia de Medellín entre el 2004 y el 2014. Se recurrió al análisis documental y a la observación participante en espacios de gestión de políticas públicas y se implementaron entrevistas semiestructuradas a funcionarios gubernamentales y no gubernamentales. La información se analizó desde tres dimensiones interdependientes: ideológica, política y administrativa. Según los resultados, las acciones intersectoriales se ven favorecidas por la adopción del enfoque de derechos, la implementación de instrumentos de política pública y la búsqueda del posicionamiento de los servicios en la comunidad; sin embargo, estos aspectos están atravesados por tensiones derivadas del intento de articular diferentes miradas, relaciones de poder y acciones administrativas.

\section{Palabras clave}

Primera infancia, política social, gestión, políticas, administración local, política gubernamental, intersectorialidad.

\section{Thesauro}

Tesauro de Ciencias Sociales de la Unesco.

\section{Para citar este artículo}

Ceballos-Higuita, L., \& Otálvaro-Castro, G. J. (2021). Tramas en la construcción de la intersectorialidad para atender la primera infancia. Revista Latinoamericana de Ciencias Sociales, Niñez y Juventud, 19(2), 1-25. https://dx.doi.org/10.116oo/rlcsnj.19.2.4873

\section{Historial}

Recibido: 09.08.2020
Aceptado: 08.02.2021
Publicado: 30.04 .2021
Información artículo

Este artículo es resultado de la investigación Proceso de construcción de las relaciones intersectoriales en el programa de atención integral a la primera infancia de Medellín —Buen Comienzo2004-2014, presentado por Lorena Ceballos-Higuita para optar al título de magíster en salud mental de la Universidad de Antioquia, 2016 (realizada entre febrero de 2014 y febrero de 2015), bajo la asesoría de Gabriel Jaime Otálvaro-Castro, profesor de la Facultad Nacional de Salud Pública. Investigación inscrita en el Centro de Investigación con el código INS 494-14. Área: sociología. Subárea: sociología. 


\section{Framework of intersectionality construction for early childhood assistance}

\section{Abstract (analytical)}

This paper presents the results of qualitative research carried out from an interpretative-historical perspective, to understand the construction of intersectoral relationships in the program of early childhood care in Medellín between 2004 and 2014. Documentary analysis and participant observation were used in public policy management spaces, and semi-structured interviews with government and non-government officials were implemented. The data was analyzed from three interdependent dimensions: ideological, political, and administrative. According to the results, intersectoral actions are favored by the adoption of the rights approach, the implementation of public policy instruments, and the search for the positioning of services in the community. However, these aspects are traversed by tensions derived from the attempt to articulate different views, power relations, and administrative actions.

Keywords

Early childhood, social policy, management policy, local administration, government policy, intersectoriality.

\section{Tramas na construção da intersetorialidade para atender a primeira infância}

\section{Resumo (analítico)}

Este artigo apresenta os resultados da pesquisa qualitativa realizada desde uma perspectiva interpretativa-histórica, para entender a construção de relações intersetoriais no programa da atenção à primeira infância de Medellín entre o 2004 e o 2014. Recorreu-se à análise documental e à observação participante em espaços de gestão de políticas públicas e implementaram-se entrevistas semiestruturadas a funcionários governamentais e não governamentais. A informação foi analisada desde três dimensões interdependentes: ideológica, política e administrativa. Segundo os resultados, as ações intersetoriais se veem favorecidas pelo uso do enfoque de direitos, a implementação de instrumentos de política pública e a procura do posicionamento dos serviços na comunidade; mesmo assim, estes aspectos estão atravessados por tensões derivadas da tentativa de articular diferentes olhares, relações de poder e ações administrativas.

\section{Palavras-chave}

Primeira infância, política social, política governamental, administração local, programa social, intersetorialidade.

\section{Información autores}

[a] Facultad Nacional de Salud Pública, Universidad de Antioquia, Colombia. Psicóloga y magíster en salud mental, Universidad de Antioquia, Colombia. iD 0000-0002-7864-598X. H5: 0. Correo electrónico:

lore861024@gmail.com

[b] Facultad Nacional de Salud Pública, Universidad de Antioquia, Medellín, Colombia. Odontólogo, Universidad de Antioquia. Magíster en educación, Universidad Pedagógica Nacional. Ph. D. en Salud Colectiva, Universidad Andina Simón Bolívar, sede Ecuador. (iD) 0000-0001-5110-7738. H5: 5 Correo electrónico: gjotalvaro@gmail.com 


\section{Introducción}

¿ a siguiente investigación — desde una observación participante, revisión docuconstrucción del proceso intersectorial en el programa Buen Comienzo propuesto por la Secretaría de Educación y la Alcaldía de Medellín.

Según reportes del Concejo de Medellín, la ciudad se ha destacado por ser pionera en la formulación de políticas públicas en Colombia, entre estas la de primera infancia, la cual se gestó a partir del programa Buen Comienzo. El programa fue creado en 2004; en 2010 obtuvo el premio ONU Hábitat, que reconoció su impacto social (Agencia Española de Cooperación Internacional para el Desarrollo, 2014) y en 2011 se adoptó como política pública.

Buen Comienzo es un programa de educación inicial adscrito a la Secretaría de Educación Municipal, dirigido a niñas y niños menores de 5 años, así como a familias gestantes y lactantes residentes en la ciudad, registradas en el Sistema de Información de Potenciales Beneficiarios (Sisbén) con un puntaje definido. ${ }^{1}$ También está dirigido a familias en situación de desplazamiento, familias en proceso administrativo de restablecimiento de derechos, familias de grupos étnicos, hijos de actores desvinculados o desmovilizados del conflicto armado y familias refugiadas en la ciudad. Esta población recibe acompañamiento en salud, nutrición, atención psicosocial, estimulación adecuada, educación inicial y recreación. Las familias gestantes y lactantes reciben acompañamiento por medio de encuentros periódico y visitas de seguimiento. Al cumplir 2 años, los niños y niñas pueden ingresar a un centro o jardín infantil, en jornadas de cinco u ocho horas al día, siendo atendidos por instituciones particulares contratadas por la Alcaldía Municipal (Secretaría de Educación de Medellín, 2020).

\footnotetext{
${ }^{1}$ Este puntaje clasifica a la población de acuerdo con sus condiciones socioeconómicas; por lo tanto, permite establecer el grado de pobreza de los hogares, con el propósito de focalizar (asignar) recursos de inversión social.
} 
De acuerdo con el reporte oficial de la Alcaldía de Medellín (2019), al finalizar el 2019 el programa contaba con más de 240 sedes en todas las comunas y corregimientos de la ciudad, brindó atención a 82650 niños y 12 ooo familias lactantes y gestantes, involucró 51 entidades aliadas y tuvo una duración de 219 días de atención por año.

Buen Comienzo constituyó un hito en las políticas sociales para la primera infancia en el país, pues esta iniciativa de protección integral se llevó a cabo en medio de un contexto normativo cuyo enfoque predominante para la atención de la infancia fue el de la restitución de derechos, incluido en el denominado Código del Menor (Presidencia de la República, 1989). Luego de dos años de ejecución del programa en la ciudad de Medellín, se aprobó el Código de Infancia y Adolescencia con un enfoque de derechos, tanto para la prevención de la vulneración de los derechos como para la promoción de sus garantías (Congreso de la República, 2006).

En la actualidad, los indicadores de vulneración de derechos de los niños, niñas y adolescentes en Medellín han aumentado, sobre todo, entre el 2016 y el 2018. En dicho periodo se registró un incremento del $30 \%$ en las violencias presentadas en el entorno familiar, tales como maltrato físico, psicológico y económico (Secretaría de Inclusión Social, Familia y Derechos Humanos, 2020). Esta situación no es particular de la ciudad de Medellín; se presenta en otras ciudades latinoamericanas (Devries et al., 2019) y se explica por el deterioro progresivo de los factores socioeconómicos en la última década (Comisión Económica para América Latina y el Caribe [Cepal], 2020). Tal deterioro ha reducido elementos esenciales de protección y se puede agravar por la actual crisis de la enfermedad del COVID-19, la que puede incrementar la vulnerabilidad y exposición de la infancia y la adolescencia a la violencia (Cepal \& Fondo de las Naciones Unidas para la Infancia [Unicef], 2020). Esto pone en duda la efectividad de las acciones orientadas a la garantía integral de los derechos, pero a la vez impulsa a seguir insistiendo en lograrlas, desde la suma de esfuerzos que hace cada sector (salud, educación, recreación, justicia).

En el 2007 se aprobó la primera política pública nacional de primera infancia, mediante el documento del Consejo Nacional de Política Económica Social ${ }^{2} 109$ (Conpes, 2007) y, luego, en el 2013, se estableció la estrategia para la atención integral de la primera infancia. En las dos iniciativas se resaltan los avances y desarrollos de la ciudad de Medellín, que tenía su propia política pública desde el 2011 (De Cero a Siempre, 2013; Departamento Nacional de Planeación, 2007). Por último, en el 2016, esa estrategia fue presentada como

${ }^{2}$ Creado por la Ley 19 de 1958 . Constituye un organismo asesor del Gobierno en todos los aspectos relacionados con el desarrollo económico y social del país. 
política pública nacional (Congreso de la República, 2016). En Medellín, en el 2015, dicha política ya había tenido una redefinición en la regulación municipal (Concejo de Medellín, 2015).

Desde un comienzo, los referentes que orientan el programa municipal se fundamentaron y apuntaron hacia la garantía y protección de los derechos de la niñez; de este modo, se hacían necesarias acciones intersectoriales, condición indispensable en la gestión de respuestas sociales con criterios de integralidad, universalidad y equidad (Cunill, 2014; Peirano, 2019). En el 2018, el gobierno de la ciudad presentó resultados de una evaluación sobre el impacto de la atención en los niños y niñas, concluyendo: «la intersectorialidad demanda, permanentemente, el desafío de construir, implementar, y evaluar programas, políticas, propuestas y escenarios educativos que nos permitan algún día superar las terribles desigualdades y erradicar el sufrimiento de nuestras niñas y niños» (Alcaldía de Medellín, 2018, p. 73); no obstante, dicho informe no presenta las estrategias, avances y desafíos de la construcción de intersectorialidad en el programa durante los catorce años de ejecución.

La gestión de respuestas sociales a favor de la primera infancia se realiza en un contexto complejo y problemático; en primer lugar, porque en dicho proceso convergen directrices locales, nacionales e internacionales que se entrelazan con lógicas y dinámicas particulares, en ocasiones contradictorias. En segundo lugar, la complejidad de la situación se debe a que se involucran decisiones de un conjunto de instituciones de las que dependen la materialización efectiva de los derechos de la infancia, sobre todo, relacionadas con el sector salud, el sistema de bienestar familiar y el sistema educativo, que presentan problemas de funcionalidad, articulación, calidad de la información, suficiencia de talento humano, entre otras (Durán-Strauch et al., 2011; Franco, 2014). Finalmente, como otro grado de complejidad, están las respuestas sociales, las cuales se dan en unas precarias condiciones de existencia y desarrollo, abarcando una proporción importante de la primera infancia de la ciudad, inmersa en un contexto de pobreza, desplazamiento forzado y violencia intrafamiliar, entre otros procesos críticos (Departamento Administrativo Nacional de Estadística, 2017; Medellín Cómo Vamos, 2018). La disonancia entre las directrices institucionales y los contextos de vida de las niñas y niños de la ciudad muestran una brecha entre el discurso político, las acciones y los resultados obtenidos.

La interacción de diferentes instituciones implicadas en la gestión de respuestas para afrontar las necesidades sociales involucra un conjunto de acciones que incluye dinámicas de planeación, implementación y reformulación de estrategias, las cuales han sido 
evidenciadas por autores como Junqueira (1998) y se han presentado en discusiones actuales como las propuestas por Zurbriggen y González (2014), quienes ven en tal necesidad de articulación un desafío para la transformación. Dichas acciones se realizan bajo las condiciones de modelos de asociación público-privado en las que se involucran aspectos ideológicos, políticos y administrativos; es decir, hay múltiples visiones, saberes y concepciones construidas frente a la población y sus situaciones (lo ideológico). En ese mismo sentido, también se conjugan las voluntades de los actores, los niveles de jerarquía según sus funciones y roles, sus intereses y expectativas, las políticas públicas (lo político) y, del mismo modo, está presente la información, los recursos materiales y humanos, la cultura organizacional y las acciones técnicas (lo administrativo) (Castell-Florit, 20o7; Cunill, 2014; Malvezzi \& Nascimento, 2020; Shankardass et al. 2012).

La intersectorialidad, como proceso de interacción entre instituciones de diferentes sectores, se construye con dos propósitos principales: por un lado, lograr una mayor eficiencia en las acciones de cada actor, desde la optimización de sus fortalezas y la limitación de sus debilidades; por otro, como lo plantea Junqueira (1998), la acción intersectorial procura generar respuestas coherentes que demandan la complejidad de las necesidades sociales de la población, en sintonía con la multicausalidad e interrelación de los problemas; aspecto en el que coinciden otros autores como Castell-Florit (2007), Zurbriggen y González (2014), Cunill (2014) y Serrano (2019).

La intersectorialidad hace referencia a un modo de construcción de interacciones en el marco de las relaciones Estado-sociedad, en oposición al concepto de sectorialidad, que remite a las lógicas de acción institucional que distinguen un tipo de actor y de actividades (como el sector público, el sector privado) o de conocimientos especializados en la organización funcional del aparato gubernamental, dispuestos para actuar con criterio de especialización en la división del trabajo, como sector educación, sector salud, sector trabajo, sector vivienda, sector agricultura, etc. (Castro, 1990; Cunill, 2014). Así, la intersectorialidad hace referencia a las relaciones entre los diversos sectores gubernamentales y diferentes modalidades de asociación entre sectores públicos y privados (Cunill, 2014).

En la última década, tanto en Colombia como en América Latina, ha aumentado el interés por comprender los aspectos involucrados en el desarrollo de la intersectorialidad. En los estudios revisados se evidencia que las políticas públicas han incluido más mecanismos para dar respuesta a las necesidades de la población desde la interacción entre sectores (Cunill et al., 2013; Chiari et al., 2018; Fiorat et al., 2017; Rezende et al., 2015). Aún así, en la implementación de estas políticas, las investigaciones han identificado 
aspectos que obstaculizan su materialización, entre ellos los desequilibrios en las relaciones de poder (Cunill et al., 2013; Chiari et al., 2018; Paulo \& Baráibar-Ribero, 2019; Zuliani et al., 2015). El trabajo de Durán-Strauch (2017) señala que un rasgo común es que las relaciones establecidas entre los sectores se dan como una suma de acciones sin lograr integralidad. Otras investigaciones, desde el análisis teórico, proponen modelos para configurar los procesos intersectoriales y resaltan la importancia de generar acciones de articulación desde los actores del nivel local (Castell-Florit \& Gispert, 2018; Malvezzi \& Nascimento, 2020); sin embargo, hace falta indagar más acerca de las barreras y facilitadores de la construcción de la intersectorialidad en prácticas sociales concretas. Por tanto, y teniendo en cuenta los desafíos pendientes de la garantía de los derechos de la infancia de la ciudad y la necesidad de avanzar en la gestión de respuestas integrales que involucran a múltiples actores sociales, este trabajo pretendió aportar conocimiento para rescatar las perspectivas y aprendizajes derivados de la práctica concreta realizada en el marco del programa de atención integral a la primera infancia Buen Comienzo de Medellín, desde una investigación que estuvo orientada por el objetivo de comprender la construcción de relaciones intersectoriales en el programa de atención a la primera infancia de Medellín entre el 2004 y el 2014.

\section{Método}

Se realizó un estudio cualitativo, siguiendo algunas de las premisas de Denzin y Lincon (2005) y de Minayo (2009), quienes sugieren una perspectiva interpretativa-histórica, en tanto que Malvezzi y Nascimento (2020), entre las características que señalan de la intersectorialidad, exponen: «es un mundo intensamente enredado en el tiempo y en el espacio» (p. 9). Se buscó entonces recapturar de este proceso un «todo-con-sentido» (Vasco, 1990, p. 11).

La recolección de información se realizó combinando tres técnicas: la observación participante, la revisión documental y las entrevistas semiestructuradas (Hernández et al., 2014). La observación participante se realizó durante diez meses en tres espacios municipales de gestión intersectorial de políticas sociales: el Comité de política pública de primera infancia, infancia y adolescencia, la Comisión de políticas públicas poblacionales y el Consejo municipal de política social para la equidad. Se realizaron apuntes en un diario de campo, en el que se registraron 25 sesiones de los espacios asistidos y en el que se destacaron situaciones y discursos que ayudaron a la comprensión del problema de investigación. 
La revisión documental incluyó diferentes fuentes, principalmente de tipo normativo y administrativo. Se revisaron actas de espacios de coordinación interinstitucional, así como también leyes, ordenanzas, acuerdos y decretos relacionados con la atención a la primera infancia; así mismo, los lineamientos técnicos para la gestión del programa en los territorios. Dichos documentos se analizaron con el apoyo de una matriz en la cual se extrajeron los elementos asociados a cada dimensión de la intersectorialidad (política, ideológica y administrativa); posteriormente, se configuró el contexto normativo, en el que se identificaron las principales orientaciones y cambios en las directrices para la acción.

Así mismo, se realizaron 13 entrevistas, con las que se pretendió comprender la subjetividad de los actores involucrados (Galeano, 2004) y «dar cuenta de las articulaciones que se dan entre las representaciones y las prácticas, ya que una cosa es lo que los sujetos sociales dicen hacer y otra es lo que hacen» (Romaní, 2013 p. 47). Para seleccionar a los entrevistados se realizó un muestreo por conveniencia, con el que se buscó representar la diversidad de actores involucrados en la gestión del programa Buen Comienzo. El muestreo fue progresivo, sujeto a la dinámica que se derivó del proceso de análisis concomitante realizado (Artiles et al., 2008). Las entrevistas fueron grabadas y transcritas de manera completa. El contenido se codificó según las categorías de análisis, de acuerdo con cada dimensión de la intersectorialidad.

Fueron sujetos del estudio personas con amplia trayectoria en la atención a la primera infancia, vinculadas a diferentes organizaciones que participaron del desarrollo del programa, tanto del sector gubernamental como no gubernamental; en este último, participaron organizaciones con y sin ánimo de lucro. Se incluyeron actores de diferentes niveles de gestión: municipal, departamental y nacional, y de diferentes sectores: salud, educación, cultura, deporte y recreación, que hubiesen participado de la gestión del programa en alguno de los periodos de gobierno (2004-2007, 2008-2011 o 2011-2014), como se puede observar en la tabla 1.

La recolección de datos se orientó por tres categorías teóricas que se conectan con dimensiones interdependientes de la construcción de relaciones intersectoriales: lo político, lo ideológico y lo administrativo. Lo político entendido como las prácticas e instituciones que gestionan el poder en torno a problemáticas sociales y que están implicadas en la construcción y reproducción de formas de ver y actuar frente a los problemas (Spinelli, 2012). Lo ideológico entendido como el conjunto de ideas, representaciones, valores y concepciones de los actores que dan sentido y sustentan la visión del mundo y la acción sobre este (Van Dijk, 2005). Por su parte, lo administrativo entendido como los procesos 
y procedimientos técnicos definidos por las organizaciones para alcanzar los objetivos trazados (Robbins \& De Cenzo, 2009). Las categorías teóricas fueron desdobladas en categorías para el análisis empírico como se presenta en la tabla 2.

\section{Tabla 1}

Características de las personas entrevistadas

\begin{tabular}{|c|c|c|c|c|c|c|}
\hline \multirow{2}{*}{ Institución } & \multicolumn{3}{|c|}{ Nivel de gestión } & \multicolumn{3}{|c|}{ Periodo de gobierno } \\
\hline & Directivo & Coordinación & Técnico & 2004-2007 & 2008-2011 & 2012-2014 \\
\hline Buen Comienzo & $x$ & & & $x$ & & \\
\hline Buen Comienzo & & & $x$ & $x$ & $x$ & $x$ \\
\hline Buen Comienzo & & & $x$ & $x$ & & \\
\hline Buen Comienzo & & & $x$ & & $x$ & \\
\hline Buen Comienzo & & & $x$ & & & $x$ \\
\hline Buen Comienzo & $x$ & & & & & $x$ \\
\hline ICBF & & $x$ & & $x$ & $x$ & $x$ \\
\hline ICBF & & $x$ & & & & $x$ \\
\hline INDER & & $x$ & & $x$ & $x$ & \\
\hline INDER & & $x$ & & $x$ & $x$ & $x$ \\
\hline Operador & & $x$ & & $x$ & $X$ & $x$ \\
\hline $\begin{array}{l}\text { Unidad de } \\
\text { Niñez }\end{array}$ & $x$ & & & $x$ & & $x$ \\
\hline Metrosalud & & & $x$ & & $x$ & \\
\hline Total & 3 & 5 & 5 & 7 & 7 & 8 \\
\hline
\end{tabular}

La información se ordenó en las categorías de análisis y en ellas se integraron las diferentes fuentes de información, lo que permitió contrastar lo que se hace, lo que se proyecta en las orientaciones normativas y lo que los actores perciben. Luego se buscaron relaciones entre las categorías, para configurar una mirada contextualizada y de conjunto del proceso estudiado. 


\section{Tabla 2}

Operacionalización de las categorías de análisis

\begin{tabular}{|c|c|c|}
\hline Dimensión & Categoría & Aspectos indagados/observados \\
\hline \multirow{6}{*}{ Política } & Actores & $\begin{array}{l}\text { Objeto social, trayectoria, acciones que realizan, roles que } \\
\text { desempeñan, cobertura. }\end{array}$ \\
\hline & Intereses & $\begin{array}{l}\text { Prioridades, temas de interés que posicionan en la agenda } \\
\text { pública de la ciudad. }\end{array}$ \\
\hline & Participación & Espacios, propósitos, compromisos públicos asumidos \\
\hline & Liderazgo & Iniciativas, propuestas, reconocimientos. \\
\hline & Conflictos & $\begin{array}{l}\text { Disensos, temas y perspectivas que suscitan situaciones } \\
\text { conflictivas. }\end{array}$ \\
\hline & Voluntad política & $\begin{array}{l}\text { Políticas que se impulsan, continuidad de las decisiones a través } \\
\text { del tiempo, disposición de recursos, así como sus criterios de } \\
\text { asignación y distribución. }\end{array}$ \\
\hline \multirow{4}{*}{ Ideológica } & $\begin{array}{l}\text { Derechos de los niños y } \\
\text { niñas }\end{array}$ & $\begin{array}{l}\text { Nociones, identificación de derechos protegidos y de alta } \\
\text { vulneración, identificación de responsabilidades con la garantía y } \\
\text { protección, explicaciones a las situaciones de vulneración. }\end{array}$ \\
\hline & Infancia & Nociones y representaciones. \\
\hline & $\begin{array}{l}\text { Contextos de vida de la } \\
\text { infancia }\end{array}$ & $\begin{array}{l}\text { Problemas que se identifican, situaciones tipo ilustrativas, expli- } \\
\text { caciones a los contextos de vida cotidiana. }\end{array}$ \\
\hline & Intersectorialidad & Nociones, valor otorgado, elementos facilitadores y obstáculos. \\
\hline \multirow{6}{*}{ Administrativa } & $\begin{array}{l}\text { Objetivos de la } \\
\text { intersectorialidad }\end{array}$ & $\begin{array}{l}\text { Objetivos declarados por los actores para disponerse en la } \\
\text { construcción de relaciones intersectoriales. }\end{array}$ \\
\hline & $\begin{array}{l}\text { Metas e indicadores en } \\
\text { relación con la intersec- } \\
\text { torialidad }\end{array}$ & $\begin{array}{l}\text { Existencia de metas e indicadores en los planes de acción de las } \\
\text { organizaciones. }\end{array}$ \\
\hline & $\begin{array}{l}\text { Procesos y procedimientos } \\
\text { que orientan la intersec- } \\
\text { torialidad }\end{array}$ & $\begin{array}{l}\text { Actividades y parámetros definidos por la organización para } \\
\text { orientar la construcción de relaciones intersectoriales, rutas de } \\
\text { atención. }\end{array}$ \\
\hline & Gestión de la información & $\begin{array}{l}\text { Criterios definidos para la gestión de la información, aplica- } \\
\text { ciones y desarrollos informáticos que soportan la gestión de la } \\
\text { información, flujo de la información, espacios de análisis. }\end{array}$ \\
\hline & Vínculos y cooperación & $\begin{array}{l}\text { Relaciones establecidas (aliados), criterios que definen la } \\
\text { búsqueda de aliados, antigüedad, beneficios reconocidos, } \\
\text { desarrollos conjuntos. }\end{array}$ \\
\hline & $\begin{array}{l}\text { Evaluación y control de la } \\
\text { intersectorialidad }\end{array}$ & $\begin{array}{l}\text { Procesos evaluativos desarrollados, medios de verificación y } \\
\text { mecanismos de control. }\end{array}$ \\
\hline
\end{tabular}

\section{Resultados}

Los hallazgos del estudio se agrupan en tres aspectos que sintetizan la experiencia de construcción de la intersectorialidad de los actores involucrados en el programa Buen Comienzo: la adopción del enfoque de derechos, la implementación de instrumentos de 
política pública y la búsqueda del posicionamiento de los servicios en la comunidad, presentándose tensiones al interior de cada uno de estos aspectos.

\section{El enfoque en derechos: un punto de partida en coexistencia con otras miradas y contradicciones}

El enfoque de derechos es el discurso que motiva la intersectorialidad en las políticas públicas de infancia en la ciudad de Medellín; esto se evidencia en el marco normativo que la ciudad ha construido para la primera infancia desde el 2004 hasta el 2015 (Concejo de Medellín, 2004, 2011, 2015). En este marco se apuesta por una política pública que, desde «el trabajo articulado, interinstitucional e intersectorial», busca que los niños y niñas tengan «desarrollo adecuado, integral, diverso e incluyente» (Concejo de Medellín, 2015, arts. 1-2). En esta misma vía, los entrevistados expresaron: «en el discurso de nosotros siempre vamos a escuchar que hacemos parte de una atención integral a la primera infancia; eso es un gran logro» (E6, 31 de octubre de 2014)․ Por su parte, la política pública nacional de primera infancia desde el 2007 (con el Conpes 109 y, luego, con la Ley 1804 de 2016) también señala que, a través del trabajo articulado e intersectorial y desde la perspectiva de derechos, se traza como objetivo garantizar la promoción y potenciación del desarrollo de los niños y niñas del país (Congreso de la República, 2016, art. 2).

Ahora bien, estos objetivos — que en definitiva son la garantía de los derechos-son interpretados de diferentes formas; estas interpretaciones se convierten en obstáculos en la consolidación de la intersectorialidad y, por lo tanto, en la materialización de dichos objetivos. En la investigación se identificaron tres filtros de interpretación:

\section{La definición de los criterios de la implementación de la política pública}

La focalización de la atención y los criterios de dicha selección de beneficiarios implica un proceso de reinterpretación de la noción de derechos por parte de los actores de la política. Aunque la política pública definió como criterios de selección de beneficiarios para la atención integral «a los niños y las niñas desde la gestación hasta cumplidos los seis (6) años inscritos en el Sisbén» (Concejo de Medellín, 2015, Art. 7), los que operan el programa señalan que «esas condiciones, por lo general, no resultan pertinentes a las realidades de las familias de los niños y niñas, lo que deja por fuera de la atención a quienes lo necesitan» (E8, 12 de noviembre de 2014). Este criterio de selección, al interpretarse

\footnotetext{
${ }^{3}$ La «e» permite identificar que esa información corresponde a una entrevista, y se presenta con la fecha en que esta se realizó.
} 
como un aspecto que se aleja del principio de universalidad del enfoque de derechos humanos, genera tensión en las relaciones entre quienes ordenan y quienes prestan de manera directa el servicio; por lo tanto, se constituye en un obstáculo para el trabajo conjunto.

La interacción con otros enfoques, especialmente la mirada soportada en la vulnerabilidad/protección

Este enfoque, desde el cual se mira a los niños y niñas como objetos de cuidado, si bien se depura en el marco normativo, persiste en las prácticas y discursos actuales de los actores involucrados en la atención. Ello fue observado en diversas discusiones en los espacios de coordinación interinstitucional, en los que se identificó que el reconocimiento de los niños y niñas como sujetos con voz y capacidad para participar en la toma de decisiones entra en conflicto con otras perspectivas que sitúan a niños y niñas como personas vulnerables y sin capacidad de tomar decisiones, producto de situaciones de vulneración de derechos y por su propia edad (O5, 29 de mayo de 2014; O6, 26 de junio de 2014). Así, la coexistencia de diferentes enfoques dificulta la construcción de acuerdos para la gestión de las respuestas.

\section{Retroalimentación positiva y efecto de bloqueo institucional}

La trayectoria de los actores crea una manera de hacer las cosas que genera un circuito de retroalimentación de lo instituido y de bloqueo a nuevos desarrollos, lo que en el análisis de política se conoce como mecanismos de retroalimentación positiva, porque refuerza la reproducción del camino elegido y crea un efecto de bloqueo institucional (Pierson, 2000). Por ello, algunos actores plantearon asuntos como: «con el código del menor de 1989, había más agilidad en la resolución de los procesos de restitución de derechos, ahora que se involucran más profesionales y entidades es más dispendioso el proceso» $\left(\mathrm{O}_{2}\right.$, 27 de marzo de 2014). De esta manera, las experiencias previas pueden sustentar una posición reactiva a la idea de gestionar las respuestas junto con otros actores, en cuanto ello implica, efectivamente, el desarrollo de procesos de mayor complejidad, lo que demanda más tiempo y exige una disposición de mayor flexibilidad y apertura a un diálogo interinstitucional permanente.

\footnotetext{
${ }^{4} \mathrm{La} \ll \mathrm{O} »$ permite identificar que esa información corresponde a una observación registrada en el diario de campo y se presenta con la fecha en que se hizo el registro.
} 


\section{Los instrumentos de la política pública: facilitadores y obstaculizadores de la acción intersectorial}

Los instrumentos de política pública definen el alcance y dirección de los programas y proyectos; así, los instrumentos de la política pública de primera infancia de Medellín ratifican a la intersectorialidad como estrategia de gestión para lograr la integralidad en la atención; en ellos se especifican los actores involucrados, sus roles y responsabilidades, las formas de financiamiento de la política y los espacios de coordinación y seguimiento. Ahora bien, esos instrumentos se construyen en medio de un contexto complejo del que no son ajenos, en los que están los intereses de cada actor, las relaciones de poder y la arquitectura institucional municipal y nacional; por eso se presentan como posibilitadores o como obstáculos para la construcción de la acción intersectorial.

En los municipios de Colombia los acuerdos municipales constituyen un instrumento de política pública de notable relevancia, pues a través de ellos se establecen las políticas de gobierno, desde el órgano legislativo municipal. En Medellín, el programa Buen Comienzo se ha respaldado en tres acuerdos municipales: en el 2004 se creó como proyecto estratégico de la Alcaldía; en el 2011 se adoptó como política pública y se amplió la población objetivo, pasando de centrarse en niños y niñas vulnerables a considerar toda la infancia de la ciudad; y en el 2015 se redefinió la política pública y se añadieron estrategias para su implementación.

Desde su adopción en 2004, el programa Buen Comienzo ha tenido continuidad en los diferentes periodos de gobierno, en los cuales se mantuvo la acción intersectorial como un eje central de la gestión del programa; no obstante, en cada periodo se introdujeron cambios que afectan la interacción entre los involucrados. Así, el Acuerdo Municipal 58 de 2011 determinó responsabilidades de los actores y definió espacios de gestión; por su parte, el Acuerdo 54 de 2015 dio relevancia a la articulación del programa con la política de infancia y adolescencia, pero modificó la especificación de los aportes de los actores involucrados y los dejó a consideración del gobierno de turno (Concejo de Medellín, 2011).

Esto sucede también con los acuerdos municipales, en los que se refleja el posicionamiento de los actores sociales que se van creando con la nueva política y se definen reglas de juego centrales del funcionamiento del programa. Estos actores precisan claramente las responsabilidades del gobierno municipal, por ejemplo, las facultades del alcalde para efectuar la asignación y los traslados presupuestales necesarios para su desarrollo 
(Concejo de Medellín, 2004, art. 4); además, obligan a precisar la financiación del programa en los planes de desarrollo municipal (Concejo de Medellín, 2011) y, a su vez, determinan que cada actor involucrado debe aportar desde sus presupuestos, según su responsabilidad en la atención, y dan vía libre a la gestión de recursos a nivel nacional, internacional y con el sector productivo. Resulta importante señalar que las normas reglamentarias definieron que no se podría disminuir el presupuesto del programa de un cuatrienio a otro (Alcaldía de Medellín, 2013, art. 37). No obstante, el Acuerdo 54 de 2015, por medio del cual se redefine la regulación municipal sobre la Política Pública de Primera Infancia (Concejo de Medellín, 2015), estableció que el presupuesto debía estar sujeto al análisis del impacto fiscal, lo cual lo cual contribuye al incumplimiento de la premisa de progresividad presupuestal inicialmente planteada, y que había sido tendencia en la primera década del siglo XXI; al parecer como medida que pretendía legalizar lo que en efecto ya venía ocurriendo en términos del incumplimiento a la premisa de financiación progresiva. El presupuesto del programa aumentó progresivamente entre 2004 y 2012, para luego disminuir durante los años 2013 y 2014.

Frente a los espacios para la coordinación y seguimiento de la política pública, estos se han reconfigurando en busca de seguir con la construcción intersectorial y reflejan el movimiento de los actores y los cambios en las dinámicas institucionales. Los espacios interinstitucionales de coordinación para la atención integral a la primera infancia son novedosos, pero aún incipientes, dado que todavía son débiles los mecanismos para propiciar una amplia y sostenida participación de la sociedad civil y de los actores privados; además, apenas inicia la articulación entre las políticas poblacionales. Este proceso de articulación, si bien se gestó en el nivel municipal, ha servido para propiciar una interacción hacia instancias nacionales. En los inicios del programa, entre el 2004 y el 2007, estos espacios de coordinación estuvieron bajo responsabilidad de la Alcaldía. Luego, se articularon al Consejo de política social para la equidad (Compse) en coherencia con los lineamientos nacionales; además de este espacio, se contaba con el Consejo de política pública de infancia y adolescencia, el cual se fusionó junto con otros espacios de seguimiento de políticas poblacionales al Compse (Congreso de la República, 2006).

Así mismo, desde el orden nacional, se creó en el 2011 la Comisión Intersectorial de Primera Infancia (Cipi) (Presidencia de la República, 2011). En el municipio se pasó de tener un espacio específico para el seguimiento de la política de primera infancia a la articulación con el comité técnico interinstitucional de política pública de infancia y adolescencia, pues ambos espacios convocaban a los mismos actores. Posteriormente, en 
consonancia con las orientaciones de la Cipi, se volvió a definir un espacio específico para el seguimiento de la política de primera infancia sin dejar la articulación con el comité de infancia (Secretaría de Educación de Medellín, 2018). Desde lo observado, se identifica que se intentó generar mecanismos de articulación entre las políticas poblacionales, a la vez que se propuso un proyecto de acuerdo municipal para formalizar dicha instancia; sin embargo, no se logró el respaldo gubernamental a la iniciativa.

\section{El posicionamiento del programa en la comunidad y en los territorios}

El programa Buen Comienzo ha buscado posicionar la política en la comunidad a partir de dos elementos: la prestación de servicios con calidad y el desarrollo de estrategias de comunicación. Para lo primero, el principal instrumento utilizado ha sido la definición de lineamientos técnicos, es decir, un mecanismo de implementación de la política pública, como lo señala Salamon (2002, como es citado en Sarthou, 2015), en este caso, en la forma de orientaciones de obligatorio cumplimiento para los actores involucrados, establecidas en términos de condiciones contractuales. Desde los lineamientos técnicos se definen de manera detallada las condiciones de la prestación de los servicios, las formas de articulación con otros actores en el territorio y el rol esperado de cada actor en el proceso de atención integral. En ese sentido, los lineamientos técnicos, como expresión de un acto administrativo municipal, proponen la relación intersectorial desde un mandato contractual y hacen visible la articulación de lo local con lo nacional.

Si bien los lineamientos tienen como objetivo tener mayor calidad en la atención a partir de la estandarización de procesos en las diferentes entidades prestadoras de servicios. Estos también pueden ser leídos como limitantes para la construcción interinstitucional, al desconocer las particularidades de cada institución prestadora, pues los lineamientos técnicos tensionan las relaciones entre los actores, particularmente desde el actor gubernamental hacia los actores particulares prestadores de servicios educativos a la primera infancia, como lo expresa uno de los actores:

Yo pienso que el municipio exige; esa puede ser su labor y tiene que hacerlo; pero a veces solicita mucha información inmediata. Otra entidad con la que tenemos otros contratos para prestar el servicio, y es del orden nacional, acepta también las dinámicas de la institución y tiene en cuenta la experiencia del operador (...). Como institución nos hemos fortalecido mucho con esas exigencias; pero si un trabajo de una institución tiene, por ejemplo, la misma propuesta y sustenta lo que pide la entidad contratante, ¿por qué no 
valerla? «es que tiene que ser así» (...). Pretender unificar, y es una discusión que tenemos también acá, no es bueno, porque los operadores tienen cosas muy bonitas de su historia, de cómo fueron creadas. (E5, 29 de septiembre de 2014)

Desde la percepción de los operadores, en los inicios del programa los prestadores tuvieron un mayor reconocimiento, ya que se les tuvo más en cuenta para la construcción de los procesos del programa, lo que fue disminuyendo a la par que ganó relevancia el papel de la academia, como actores delegados por el ente gubernamental para darle sustento teórico a las especificaciones técnicas de los lineamientos. En contraste, en el orden nacional, se plantea una perspectiva desde la cual a los prestadores del servicio son aliados estratégicos en la gestión, «reconociendo en cada uno de los actores y aliados estratégicos las experiencias, recursos, la capacidad instalada, los saberes» (Instituto Colombiano de Bienestar Familiar, 2019, p. 46). Emerge así una tensión en la construcción de relaciones intersectoriales relativa al modo de institucionalización y gestión del programa, realizada a través de terceros, como si de ello dependiera la mirada del ente gubernamental, dando paso de una mirada de los prestadores como socios o aliados estratégicos a una en que a mayor grado de institucionalización se les define en términos de agentes contractuales, la cual podría limitar la apertura para su consideración como un agente activo o socio clave de la construcción de la intersectorialidad.

Frente al papel de las estrategias comunicacionales, los actores reconocen dos tipos: el primero está orientado a generar impacto a nivel de ciudad; se realiza bajo la modalidad de feria o festival anual; ha logrado congregar a miles de ciudadanos y familias participantes del programa en cada versión; y, en él, la orientación y liderazgo es de la Alcaldía municipal, aunque son los operadores los encargados de hacer el montaje y el desarrollo de las actividades. Por otro lado, en el segundo papel los actores reconocen las estrategias comunicacionales territoriales, especialmente desplegadas por las instituciones con presencia en el territorio y en contacto directo con las familias, que deben seguir el protocolo dado por el ente gubernamental. Las estrategias comunicacionales se convierten en un aspecto que tensiona las relaciones entre los actores, sobre todo por su orientación vertical y los problemas de reconocimiento y legitimación social que producen, aunque posicionan de manera especial al actor gubernamental e invisibilizan a los actores locales y particulares. 


\section{Discusión}

La multiplicidad de actores puede representar una ventaja potencial porque se pueden generar dinámicas de complementariedad (Cunill, 2014; Junqueira, 1998; Repetto, 2006) y con ellas aumentar el alcance de las respuestas a las complejas situaciones de vida que enfrenta la primera infancia; no obstante, esto se limitado por las características de los modelos de gestión implementados y por la falta de consistencia en la apuesta de construcción de la acción intersectorial (figura 1).

\section{Figura 1}

La intersectorialidad como proceso: actores, pasos y dimensiones para su configuración

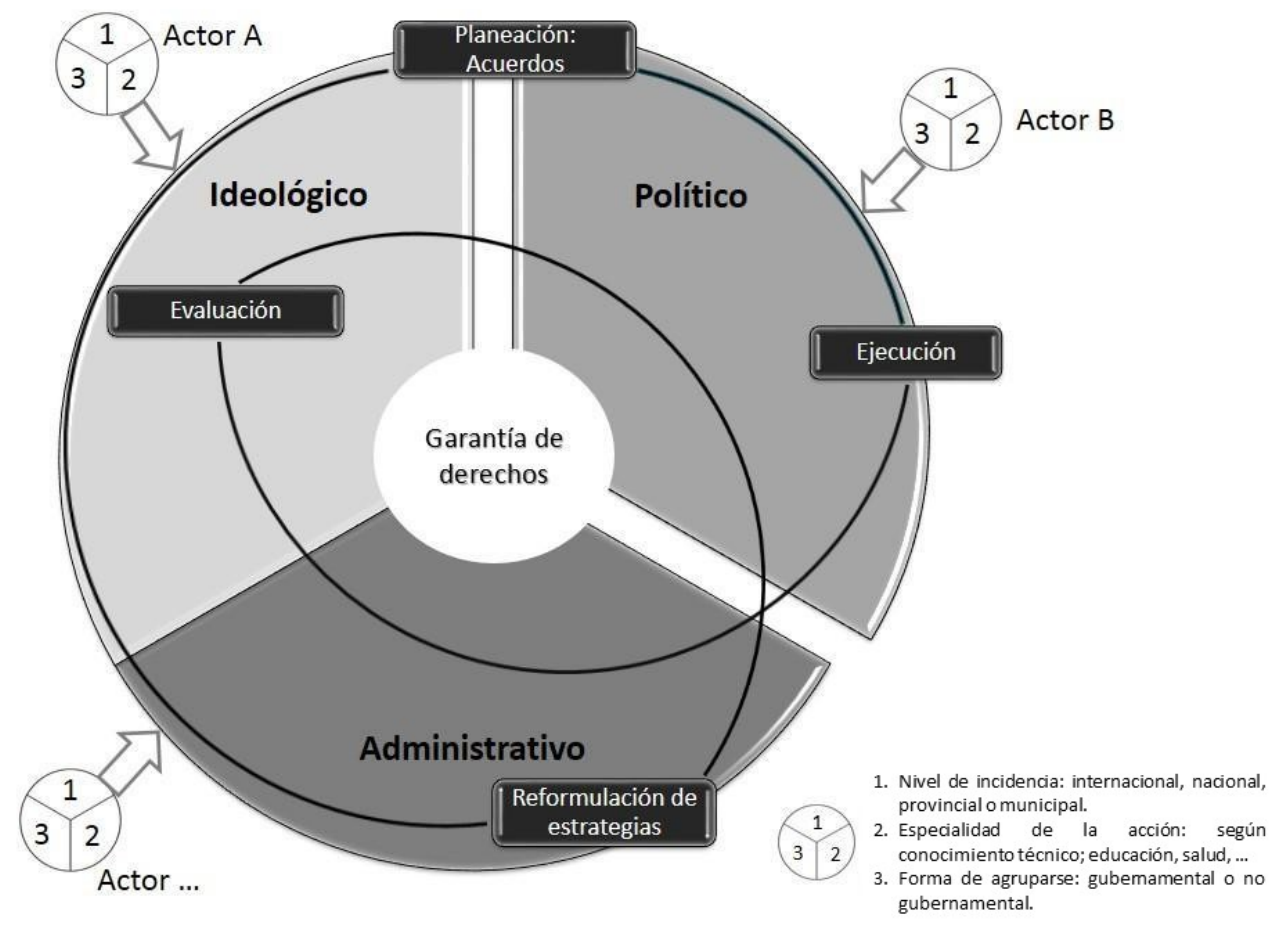

Nota. Basada en los aportes de Castell-Florit (2007), Cunill (2014); Junqueira (1998) y Repetto (2006).

Ahora bien, lograr esa complementariedad implica un proceso en el cual se interactúa con múltiples actores de diferentes características, que están dadas por su nivel de incidencia (internacional, nacional, provincial o municipal), por la especialidad en su accionar (según su conocimiento técnico y la población en la que se centra) y por la naturaleza de la organización (gubernamental o no gubernamental). Además, comprende lo que 
Junqueira (1998) denomina un «ritmo dinámico» (p.3), de avances y retrocesos, en el que se puede estar en dirección de construcciones nuevas, o en dirección de retomar o reformular lo hecho. De esta manera, los procesos de construcción de la intersectorialidad atraviesan momentos de planeación, ejecución, evaluación y reformulación de estrategias, no se desarrollan de manera lineal, incluso, no todos se dan. Sumado a ello, la direccionalidad de dichos procesos está permeada por aspectos ideológicos, políticos y administrativos, que configuran un entramado dinámico y conflictivo.

Si bien el enfoque de derechos ha permitido una resignificación de la mirada y de las acciones (Cunill, 2014) (por la concepción que da de los niños y niñas como sujetos de derechos, por especificar esos aspectos mínimos que deben ser garantizados para un desarrollo digno y porque se señala los responsables de esas garantías), se presentan diferentes apropiaciones en los actores según los intereses, las tradiciones institucionales y la misionalidad de ellos; lo que lleva a plantear diferentes maneras de concretar su rol en la garantía de los derechos. Esto podría explicar la persistencia de una mirada fragmentada de la niñez (Cursino \& Fujimori, 2012), en la que cada actor involucrado en la atención se centra en reconocer lo que le corresponde brindar y en minimizar la necesidad de interactuar con otros para dar respuesta integral a las necesidades de niños y niñas.

Por lo tanto, la construcción de la intersectorialidad implica un cambio de visión, como lo dice Junqueira (1998), y el cambio se daría en la medida en que se dejan de lado las posiciones radicales; solo así se superarían los absolutismos (Paulo \& Baráibar-Ribero, 2019; Rezende et al., 2015; Zuliani et al., 2015).

Por otro lado, como se ha reiterado en diferentes investigaciones (Cunill et al., 2013; Chiari et al., 2018; Fiora et al., 2017; Rezende et al., 2015) y se observa en el presente estudio, las políticas públicas viabilizan las acciones intersectoriales, por lo que constituyen un medio para su desarrollo (Cunill, 2014). Sin embargo, su construcción y su implementación, en un contexto de amplia tercerización, están mediados por relaciones conflictivas, que se configuran por la trama de ideas, relaciones de poder y formas de organización y gestión, en lo que cada actor propende hacia la defensa de sus intereses - como actor de mercado- y cuya respuesta integral a las necesidades sociales de la población puede quedar relegada a un segundo plano, reproduciendo con ello un modo de gestión abierto a múltiples actores, pero de alta dispersión (Castell-Florit \& Gispert, 2018; Rezende et al., 2015).

En el camino para la implementación de esas políticas públicas, que proponen la intersectorialidad como mecanismo clave de su desarrollo, también se evidencian los obstáculos que se presentan en la coordinación entre niveles de gestión nacional y municipal (Abello \& 
Acosta, 2006), así como los deficientes mecanismos para garantizar una participación deliberativa y con incidencia de la sociedad civil, lo que produce un efecto que se ve reflejado en la debilidad de las acciones locales comunitarias (Chiari et al., 2018; Duarte et al., 2011; Fiorat et al., 2017; Jhons, 2010; Paulo \& Baráibar-Ribero, 2019; Zuliani et al., 2015).

$\mathrm{Al}$ observar en conjunto las barreras para la construcción de la intersectorialidad, se puede identificar que gran parte de estas se relacionan directamente con una implementación de políticas públicas basadas en modelos de asociación público-privado. En estos últimos, mayoritariamente se utiliza la tercerización como mecanismo de construcción de alianzas, que están mediadas por los recursos económicos y los nexos políticos de los actores involucrados (Álvarez et al., 2019).

Como se identificó en el presente estudio, la acción gubernamental es un claro eje dinamizador de la construcción de la intersectorialidad, ya que a través de esta se torna posible la construcción y puesta en marcha de políticas públicas (Abello \& Acosta, 2006; Cunill, 2014); no obstante, también desde allí se originan muchos de los obstáculos señalados. De ahí que se señale también la posibilidad de cualificar la apuesta por el trabajo intersectorial que está sustentada de manera más firme en la fuerza de la voluntad política y el liderazgo de actores con poder de convocatoria; esto son capaces de dinamizar espacios de diálogo y reconocimiento de saberes, perspectivas y experiencias de los actores locales involucrados en torno a un problema u objetivo común y no solo como un asunto de autoridad (Otálvaro-Castro \& López, 2017). Por ello, Castells-Florit y Gispert (2018) señalan la importancia del fortalecimiento de los liderazgos sociales, comunitarios y no gubernamentales, así como la necesidad de una formación que promueva una cultura cooperativa, el trabajo en equipo y habilidades de negociación (Fiorat et al., 2017; Rezende et al., 2015).

Finalmente, una de las limitaciones del estudio puede ser la existencia de un sesgo en la formación, que se deriva de los datos autoinformados (Avello et al., 2019) ya que al indagar por un proceso que en su momento llevaba diez años de ejecución, en el cual los participantes habían estado en diferentes periodos de tiempo, se pudo presentar memoria selectiva y también el efecto telescopio, en los que los informantes recuerdan eventos que ocurrieron una vez como si ocurrieran en otro tiempo. Por otro lado, se pudieron presentar sesgos de atribución, porque los participantes, los entrevistados y quienes hacían parte de los espacios de observación, al representar una institución, pudieron atribuir eventos positivos y resultados a la propia institución, pero atribuir eventos negativos y resultados a 
fuerzas externas. Finalmente, se pudo dar exageración, que es el acto de representar resultados o embellecer eventos como más significativos de lo que realmente fueron.

\section{Conclusiones}

La construcción de relaciones intersectoriales en las políticas públicas está mediada por los diferentes aspectos en que se entrelazan ideas, relaciones políticas y formas de organización y gestión de las respuestas sociales. El reconocimiento de ello da luces para comprender la complejidad de la construcción de la intersectorialidad y valorar los avances y las barreras para su materialización en prácticas sociales concretas.

En el caso de la atención integral a la primera infancia en Medellín, la construcción de relaciones intersectoriales está fuertemente influenciada y se ve favorecida por la adopción explícita de un enfoque de derechos como orientador de la política pública, así como por la construcción de un soporte normativo y de instrumentos técnicos en los que se definen los roles de los actores y espacios de coordinación con una vocación intencionada hacia el trabajo conjunto entre los múltiples actores involucrados. Los actores gubernamentales han sido una pieza clave de esto.

No obstante, el entramado de relaciones se configura en un contexto de fuertes tensiones, que limitan el despliegue del potencial del trabajo intersectorial en favor de la garantía de los derechos de los niños y niñas de la ciudad. Particularmente, estas tensiones derivan de la inconsistencia de la apuesta gubernamental por el enfoque de derechos respecto a las limitaciones de la universalidad de la cobertura (mientras que el criterio de focalización resulta predominante), la timidez para materializar una perspectiva de ciudadanía infantil (en tanto la mirada desde la vulnerabilidad continúa siendo la predominante), así como por la generación de un efecto de bloqueo institucional a la perspectiva emergente de derechos y la permanencia de modelos comunicativos y de gestión en los que prevalece el posicionamiento unilateral del actor gubernamental en detrimento del reconocimiento y legitimación social del conjunto de actores implicados en la construcción intersectorial del programa de atención a la primera infancia.

Un aspecto nodal que explica una parte importante del entramado de barreras que limitan un mejor desarrollo de la acción intersectorial en el programa de atención integral a la primera infancia de Medellín está directamente relacionado con la adopción de un modelo de gestión basado en la tercerización; en este la calidad de las relaciones entre 
los actores depende de manera preponderante de los recursos de poder que movilizan los involucrados. La superación de estas barreras pasa por la capacidad de los actores de reflexionar críticamente las implicaciones del modelo de gestión dominante y repensar otros modelos posibles de relacionamiento entre la sociedad y el Estado.

\section{Referencias}

Abello, R., \& Acosta, A. (2006). Recomendaciones para la política pública de primera infancia en materia de educación inicial a partir del estudio de cinco modalidades de atención a la primera infancia en Bogotá, Colombia. Journal of Education for International Development, 2(3).

Agencia Española de Cooperación Internacional para el Desarrollo. (2014). Cooperación descentralizada y la eficacia de la ayuda. n.. 2 : Medellín. Una mirada desde los gobiernos locales de América Latina. https://bit.ly/zolwDcq

Alcaldía de Medellín. (2013). Por el cual se reglamenta el acuerdo 58 de 2011, mediante el cual se adoptó la política pública de Atención Integral a la Primera Infancia Buen Comienzo, se desarrolló un Sistema de Atención Integral y se modificó el Acuerdo 14 de 2004. [Decreto 1277 de 2013]. GO: 4176. Medellín.

Alcaldía de Medellín. (2018). Efectos del programa buen comienzo en el desarrollo integral de niños y niñas que participan de la modalidad institucional 8 horas en centros y jardines infantiles de la ciudad de Medellín 2018. https://bibliotecadigital.udea.edu.co/ bitstream/10495/14770/1/BarretoMesaMaribel_2019_EfectosBuenComienzo.pdf

Alcaldía de Medellín. (2019). Buen Comienzo celebra sus 15 años con un nuevo jardín infantil. Alcaldía de Medellín. https://especiales.semana.com/regionales/medellin/buencomienzo-programa-para-la-primera-infancia-en-medellin.html

Álvarez, L., Goez, J., \& Quintero, M. (2019). The public-private partnership model in the management of early childhood policy in Medellín: Case study. Revista Gerencia y Políticas de Salud, 18(36). https://doi.org/10.11144/javeriana.rgps18-36.pppm

Artiles, L., Otero, J., \& Barrios, I. (2008). Metodología de la investigación para las Ciencias de la Salud. Editorial La Habana.

Avello, R., Rodríguez, M., Rodríguez, P., Sosa, D., Companioni, B., \& Rodríguez, R. (2019). ¿Por qué enunciar las limitaciones del estudio? MediSur, $17(1)$, 10-12.

Castell-Florit, F. (2007). Comprensión conceptual y factores que intervienen en el desarrollo de la intersectorialidad. Revista Cubana Salud Pública, 33(2), 1-13. https://doi.org/gch2 
Castells-Florit, F., \& Gispert, E. (2018). Empoderamiento intersectorial en directivos y líderes locales como contexto para la acción en salud pública. Revista Cubana de Salud Pública, 44(3), 1-10.

Castro, G. (1990). Las políticas sociales integradas en la perspectiva de la intersectorialidad y de la acción de la Unesco en apoyo a las estrategias de desarrollo para América Latina y el Caribe en el marco del ajuste. Revista Educación Superior y Sociedad, 1(1), 89-96.

Comisión Económica para América Latina y el Caribe. (2020). América Latina y el Caribe ante la pandemia del COVID-19: efectos económicos y sociales. Informe especial COVID-19 $n .{ }^{\circ}$. https://doi.org/10.18356/9789210054720

Comisión Económica para América Latina y el Caribe, \& Fondo de las Naciones Unidas para la Infancia (2020). Violencia contra niñas, niños y adolescentes en tiempos de COVID-19. https://bit.ly/3bqhqkM

Concejo de Medellín. (2004). Por medio del cual se crea el programa «Buen Comienzo» para promover el desarrollo integral, diverso e incluyente de los menores de 6 años en la ciudad de Medellín. [Acuerdo 14 de 2004]. GO: 2264. Autor.

Concejo de Medellín. (2011). Por medio del cual se adopta la política pública de atención integral a la primera infancia Buen Comienzo, se desarrolla un Sistema de Atención Integral y se modifica el Acuerdo 14 de 2004. [Acuerdo 58 de 2011]. GO: 3975. Autor.

Concejo de Medellín. (2015). Por medio del cual se redefine la regulación municipal sobre la política pública de primera infancia y el programa Buen Comienzo. [Acuerdo 54 de 2015]. GO: 4356. Autor.

Congreso de la República [Colombia]. (2006). Deroga el Decreto 2737 de 1989. Por la cual se expide el Código de la Infancia y la Adolescencia. [Ley 1098 de 2006]. DO: 46.446. Autor.

Congreso de la República [Colombia]. (2016). Por la cual se establece la política de Estado para el desarrollo integral de la primera infancia de Cero a Siempre y se dictan otras disposiciones. [Ley 1804 de 2016]. DO: 49.953. Autor.

Consejo Nacional de Política Económica y Social. (2007). Política pública nacional de primera infancia «Colombia por la Primera Infancia». https://bit.ly/3waCPX 5

Cunill, N. (2014). La intersectorialidad en las nuevas políticas sociales: un acercamiento analítico-conceptual. Gestión y Política Pública, 23(1), 5-46.

Cunill, N., Fernández, M., \& Thezá, M. (2013). La cuestión de la colaboración intersectorial y de la integralidad de las políticas sociales: lecciones derivadas del caso del sistema de protección a la infancia en Chile. Polis, 12(36), 289-314. https://doi.org/gjsj84 
Cursino, E., \& Fujimori, E. (2012). Integralidad como una dimensión de las prácticas de atención a la salud del niño: una revisión bibliográfica. Revista Enfermagem UERJ, 20(1), 676-680.

Chiari, A., Ferreira, R., Akerman, M., Amaral, J., Machado, K., \& Senna, M. (2018). Rede intersetorial do Programa Saúde na Escola: sujeitos, percepções e práticas. Cadernos de Saúde Pública, 34(5), e00104217. https://doi.org/10.1590/0102-311X00104217

De Cero a Siempre. (2013). Estrategia de atención integral a la primera infancia: fundamentos políticos, técnicos y de gestión. https://bit.ly/3bsj71a

Denzin, N. K., \& Lincoln Y. S. (2005). The Sage handbook of qualitative research. Sage.

Departamento Administrativo Nacional de Estadística. (2017). Pobreza monetaria y multidimensional en Colombia 2017. https://bit.ly/3uKAimi

Departamento Nacional de Planeación [Colombia]. (2007). Política pública nacional de primera infancia "Colombia por la primera infancia». Documento Conpes Social, 109. Ministerio de la Protección Social; Ministerio de Educación Nacional; Instituto Colombiano de Bienestar Familiar.

Devries, K., Merrill, K., Knight, L., Bott, S., Guedes, A., Butron-Riveros, B., Hege, C., Petzold, M., Cappa, C., Maxwell, L., Williams, A., Kishor, S., \& Abrahams, N. (2019). Violence against children in Latin America and the Caribbean: What do available data reveal about prevalence and perpetrators? Revista Panamericana de Salud Pública, 43, e66. https://doi.org/10.26633/RPSP.2019.66

Duarte, J., Gallego, T., \& Parra, P. (2011). Análisis de las investigaciones sobre la atención y el desarrollo de la primera infancia. Revista Latinoamericana de Ciencias Sociales, Niñez y Juventud, 9(2), 65-103.

Durán-Strauch, E. (2017). Derechos de niños y niñas: del discurso a la política local. Revista Latinoamericana de Ciencias Sociales, Niñez y Juventud, 15(2), 879-891.

Durán-Strauch, E., Guáqueta, C., \& Torres, A. (2011). Restablecimiento de derechos de niños, niñas y adolescentes en el sistema nacional de bienestar familiar. Revista Latinoamericana de Ciencias Sociales, Niñez y Juventud, 9(2), 549-559.

Fiorat, R., Serrano, P., Segura del pozo, P., \& Ramasco, M. (2017). Intersectorialidad y participación social como políticas de afrontamiento para las desigualdades en salud en el mundo. Elsevier.

Franco, A. (2014). Sistemas de salud en condiciones de mercado: las reformas del último cuarto de siglo. Revista Facultad Nacional de Salud Pública. 32(1), 95-107.

Galeano, M. E. (2004). Diseño de proyectos en la investigación cualitativa. Universidad Eafit. 
Hernández R., Fernández C., \& Baptista M. (2014). Metodología de la investigación. McGrawHill.

Instituto Colombiano de Bienestar Familiar. (2019). Lineamiento técnico para la atención a la primera infancia. https://bit.ly/3hsbKec

Jhons, S. (2010). Early childhood service development and intersectoral collaboration in rural Australia. Australian Journal of Primary Health, 16(1), 40-46. https://doi.org/dvv5g6

Junqueira, L. (1998). Descentralización, intersectorialidad y red en la gestión de la ciudad. Revista del Clad Reforma y Democracia, 12, 89-108.

Malvezzi, C., \& Nascimento, J. (2020). A teoria ator-rede e o estudo da intersetorialidade nas políticas públicas. Interface-Comunicação, Saúde, Educação, 24. https://doi.org/gch3

Medellín Cómo Vamos. (2018). ¿Cómo va la Primera Infancia en Medellín?, 2017. https:// bit.ly/3hzepCy

Minayo, M. (2009). La artesanía de la investigación cualitativa. Lugar Editorial.

Otálvaro-Castro, G. J., \& López, A. (2017). La acción intersectorial por la salud: una vía para la incidencia en los procesos sociales determinantes de la salud. En Universidad de Antioquia (Ed.), Gestión territorial de la salud: perspectivas, aprendizajes y aportes a la práctica (pp. 79-94). Universidad de Antioquia.

Paulo, L., \& Baráibar-Ribero, X. (2019). Tramas fallidas en la intersectorialidad de la política asistencial uruguaya. Prospectiva, (27), 55-82. https://doi.org/gch4

Peirano, C. (2019). Un marco de referencia para las políticas de niñez y adolescencia en América Latina y el Caribe. Revista Iberoamericana de Ciencia, Tecnología y Sociedad, $14(42), 153-172$.

Pierson, P. (2000). Increasing returns, path dependence, and the study of politics. The American Political Science Review, 94(2), 251-267. https://doi.org/10.2307/2586011

Presidencia de la República [Colombia]. (1989). Código del menor. Por medio de la cual se expide el Código General del Proceso y se dictan otras disposiciones. [Decreto 2737 de 1989]. DO: 48.489 . Autor.

Presidencia de la República [Colombia] (2011). Por el cual se crea la Comisión Intersectorial para la Atención Integral de la Primera Infancia - AIPI-y la Comisión Especial de Seguimiento para la Atención Integral a la Primera Infancia. [Decreto 4875 de 2011]. DO: 48.291. Autor.

Repetto, F. (2006). La dimensión política de la coordinación de programas y políticas sociales: una aproximación teórica y algunas referencias prácticas en América Latina. Indes. 
Rezende, M., Baptista, T., \& Amâncio-Filho, A. (2015). O legado da construção do sistema de proteção social brasileiro para a intersetorialidade. Trabalho, Educação e Saúde, 13 (2), 301-322. https://doi.org/10.1590/1981-7746-sipooo11

Robbins, S., \& DeCenzo, D. (2009). Fundamentos de la administración: conceptos esenciales y aplicaciones. Pearson Educación.

Romaní, O. (2013). Etnografía, metodologías cualitativas e investigación en salud: un debate abierto. Publicacions Universitat Rovira i Virgili. https://doi.org/gch5

Sarthou, N. (2015). Los instrumentos de política como enfoque de análisis de los sistemas de pago al mérito: contribuciones analíticas a partir del caso argentino. Perfiles Educativos, 37(149), 150-168. https://doi.org/10.22201/iisue.24486167e.2015.149.53130

Secretaría de Educación de Medellín. (2018). En Medellín, ciudad del aprendizaje, estamos unidos por la primera infancia. Boletín El Megáfono, 39.

Secretaría de Educación de Medellín. (2O20). Programa Buen Comienzo. https://bit.ly/2RSHSwD Secretaría de Inclusión Social, Familia y Derechos Humanos. (2020). Diagnóstico situacional de niños, niñas y adolescentes. Alcaldía de Medellín. https://bit.ly/zwaoWrU

Serrano, M. (2019). Intersetorialidade, a chave para enfrentar as desigualdades sociais em saúde. Revista Latino-Americana de Enfermagem, 27, e3124. https://doi.org/gch6

Shankardass, K., Solar, O., Murphy, K., Greaves, L., \& O'Campo, P. (2012). A scoping review of intersectoral action for health equity involving governments. International Journal of Public Health, 57(1), 25-33. https://doi.org/10.1007/s00038-011-0302-4

Spinelli, H. (2012). El proyecto político y las capacidades de gobierno. Salud Colectiva, 8 , 107-130. https://doi.org/10.1590/s1851-82652012000200002

Van Dijk, T. (2005). Ideología y análisis del discurso. Revista Internacional de Filosofía y Teoría social, $10(29), 9-36$.

Vasco, C. (1990). Tres estilos de trabajo en las ciencias sociales: comentarios a propósito del artículo «Conocimiento e interés» de Jürgen Habermas. Centro de Investigación y Educación Popular.

Zuliani, A., Bastidas, A., Miriam, A., \& Ariza, G. (2015). La participación: determinante social en la construcción de políticas públicas. Revista Facultad Nacional de Salud Pública, 33(1), 75-84.

Zurbriggen, C., \& González, M. (2014). Innovación y co-creación: nuevos desafíos para las políticas públicas. Revista de Gestión Pública 3(2), 329-361. https://doi.org/gch7 